\title{
Interval-valued functional differential equations under dissipative conditions
}

\author{
Nguyen Dinh $\mathrm{Phu}^{1 *}$, Truong Vinh $\mathrm{An}^{2}$, Ngo Van $\mathrm{Hoa}^{3}$ and Nguyen Thi Hien ${ }^{3}$
}

\section{"Correspondence:}

ndphu_dhtn@yahoo.com.vn

${ }^{1}$ Faculty of Mathematics and Computer Science, University of

Science, VNU, Ho Chi Minh City, Vietnam

Full list of author information is available at the end of the article

\begin{abstract}
A new concept of inner product on the space of compact convex subsets of $\mathbb{R}$ is introduced. Using this inner product we investigate the global existence and uniqueness of the solution of interval-valued functional differential equations. We apply these results to interval differential equations with distributed delays.
\end{abstract}

MSC: $34 \mathrm{KO} 05 ; 34 \mathrm{~K} 30 ; 47 \mathrm{G} 20$

Keywords: interval-valued functional differential equations; second type Hukuhara derivative; dissipative condition

\section{Introduction}

Functional differential equations (or, as they are also called, delay differential equations) play an important role in an increasing number of system models in biology, engineering, physics, and other sciences. There exists an extensive literature dealing with functional differential equations and their applications. We refer to the monographs [1-7], and references therein.

The set-valued differential and integral equations are an important part of the theory of set-valued analysis, and they have high value for control theory and its application. They were first studied in 1969 by De Blasi and Iervolino [8]. Recently, set-valued differential equations have been studied by many scientists due to their applications in many areas. For many results in the theory of set-valued differential and integral equations, the readers are referred to the following books and papers [1, 9-17] and references therein. The interval-valued analysis and interval-valued differential equations (IDEs) are special cases of set-valued analysis and set-valued differential equations, respectively. In many cases, when modeling real-world phenomena, information about the behavior of a dynamical system is uncertain and one has to consider these uncertainties to gain better understanding of the full models. The interval-valued differential can be used to model dynamical systems subject to uncertainties. The papers [18-21] are focused on the interval-valued differential equations. These equations can be studied with a framework of the Hukuhara derivative [22]. However, it causes the solutions to have increasing lengths of their values. Stefanini and Bede [18] proposed to consider the so-called strongly generalized derivative of interval-valued functions. The interval-valued differential equations with this derivative can have solutions with decreasing lengths of their values. Some very important extensions of the interval-valued differential equations are the set differential equations $[1,6$,

C2014 Phu et al.; licensee Springer. This is an Open Access article distributed under the terms of the Creative Commons Attribution License (http://creativecommons.org/licenses/by/2.0), which permits unrestricted use, distribution, and reproduction in any medium, provided the original work is properly cited. 
$12-15,19,23-32]$, the fuzzy differential equations [10,11, 33-45], and the fuzzy stochastic differential equations [46-51].

In the papers $[18,20,21]$ one can find the studies on interval-valued differential equations under generalized Hukuhara differentiability, i.e., equations of the form

$$
D_{H}^{g} X(t)=F(t, X(t)), \quad X\left(t_{0}\right)=X_{0} \in K_{C}(\mathbb{R}), \quad t \in\left[t_{0}, t_{0}+p\right],
$$

where $D_{H}^{g}$ is understood as the classical Hukuhara derivative and then as the second type Hukuhara derivative. The existence and uniqueness of a Cauchy problem is then obtained under the assumption that the coefficients satisfy a condition with the Lipschitz constant (see [18]). The proof is based on an application of the Banach fixed point theorem. In [21], under the generalized Lipschitz condition, Marek T Malinowski obtained the existence and uniqueness of solutions to both kinds of IDEs. In this paper, we study two kinds of solutions to IFDEs. The different types of solutions to IFDEs are generated by the usage of two different concepts of interval-valued derivative. Furthermore, in [4], Lupulescu established local and global existence and uniqueness results for fuzzy functional differential equations. Malinowski [6] studied the existence and uniqueness result of the solution to the delay set-valued differential equations under the condition that the right-hand side of the equation is Lipschitzian in the functional variable. Our direction of research is motivated by the results of Lupulescu [4, 5], Stefanini and Bede [18], and Malinowski [6, 20, 21, 52].

By using the concept of inner product on the fuzzy space $\left(E^{n}, D\right)$ which was introduced and studied in [5], we establish a new concept of inner product on the space $\left(K_{C}(\mathbb{R}), H\right)$ (space of compact convex subsets of $\mathbb{R}$ ). With the help of this inner product we formulate some dissipative conditions for interval-valued functional differential equations and, under these conditions, we prove the global existence and uniqueness of the solution. The paper is organized as follows. Section 2 is devoted to the preliminaries of interval-valued analysis and interval-valued differential equations. In Section 3, we formulate the notion of inner product on $K_{C}(\mathbb{R})$ and its properties. The global existence of a solution of IFDE is investigated in Section 4. In the last section, we give some examples being simple illustrations of the theory of the interval-valued functional differential equation.

\section{Preliminaries}

Let $K_{C}(\mathbb{R})$ denote the family all nonempty, compact, and convex subsets of $\mathbb{R}$. The addition and scalar multiplication in $K_{C}(\mathbb{R})$ we define as usual, i.e. for $A, B \in K_{C}(\mathbb{R}), A=[\underline{A}, \bar{A}]$, $B=[\underline{B}, \bar{B}]$, where $\underline{A} \leq \bar{A}, \underline{B} \leq \bar{B}$, and $\lambda \geq 0$, we have

$$
A+B=[\underline{A}+\underline{B}, \bar{A}+\bar{B}], \quad \lambda A=[\lambda \underline{A}, \lambda \bar{A}] \quad(-\lambda A=[-\lambda \bar{A},-\lambda \underline{A}]) .
$$

Furthermore, let $A \in K_{C}(\mathbb{R}), \lambda_{1}, \lambda_{2}, \lambda_{3}, \lambda_{4}, \in R$, and $\lambda_{3} \lambda_{4} \geq 0$, then we have $\lambda_{1}\left(\lambda_{2} A\right)=$ $\left(\lambda_{1} \lambda_{2}\right) A$ and $\left(\lambda_{3}+\lambda_{4}\right) A=\lambda_{3} A+\lambda_{4} A$. Let $A, B \in K_{C}(\mathbb{R})$ as above. Then the Hausdorff metric $H$ in $K_{C}(\mathbb{R})$ is defined as follows:

$$
H[A, B]=\max \{|\underline{A}-\underline{B}|,|\bar{A}-\bar{B}|\} .
$$


It is well known that $\left(K_{C}(\mathbb{R}), H\right)$ is a complete, separable, and locally compact metric space. We define the magnitude and the length of $A \in K_{C}(\mathbb{R})$ by

$$
H[A,\{0\}]=\|A\|=\max \{|\underline{A}|,|\bar{A}|\}, \quad \operatorname{len}(A)=\bar{A}-\underline{A},
$$

respectively, where $\{0\}$ is the zero element of $K_{C}(\mathbb{R})$, which is regarded as one point.

The Hausdorff metric (2.1) satisfies the following properties:

$$
\begin{aligned}
& H[A+C, B+C]=H[A, B] \text { and } H[A, B]=H[B, A], \\
& H[A+B, C+D] \leq H[A, C]+H[B, D], \\
& H[\lambda A, \lambda B]=|\lambda| H[A, B],
\end{aligned}
$$

for all $A, B, C, D \in K_{C}(\mathbb{R})$ and $\lambda \in R$. Let $A, B \in K_{C}(\mathbb{R})$. If there exists an interval $C \in K_{C}(\mathbb{R})$ such that $A=B+C$, then we call $C$ the Hukuhara difference of $A$ and $B$. We denote the interval $C$ by $A \ominus B$. Note that $A \ominus B \neq A+(-) B$. It is well known that $A \ominus B$ exists in the case len $(A) \geq \operatorname{len}(B)$. Besides that, we can see the following properties for $A, B, C, D \in K_{C}(\mathbb{R})$ (see $[20])$ :

- If $A \ominus B, A \ominus C$ exist, then $H[A \ominus B, A \ominus C]=H[B, C]$.

- If $A \ominus B, C \ominus D$ exist, then $H[A \ominus B, C \ominus D]=H[A+D, B+C]$.

- If $A \ominus B, A \ominus(B+C)$ exist, then there exist $(A \ominus B) \ominus C$ and $(A \ominus B) \ominus C=A \ominus(B+C)$.

- If $A \ominus B, A \ominus C, C \ominus B$ exist, then there exist $(A \ominus B) \ominus(A \ominus C)$ and $(A \ominus B) \ominus(A \ominus C)=C \ominus B$.

Definition 2.1 We say that the interval-valued mapping $F:[a, b] \subset R^{+} \rightarrow K_{C}(\mathbb{R})$ is continuous at the point $t \in[a, b]$ if for every $\varepsilon>0$ there exists $\delta=\delta(t, \varepsilon)>0$ such that, for all $s \in[a, b]$ such that $|t-s|<\delta$, one has $H[F(t), F(s)] \leq \varepsilon$.

The strongly generalized differentiability was introduced in [18] and studied in [20, 4143].

Definition 2.2 Let $X:(a, b) \rightarrow K_{C}(\mathbb{R})$ and $t \in(a, b)$. We say that $X$ is strongly generalized differentiable at $t$ if there exists $D_{H}^{g} X(t) \in K_{C}(\mathbb{R})$ such that

(i) for all $h>0$ sufficiently small, $\exists X(t+h) \ominus X(t), \exists X(t) \ominus X(t-h)$ and

$$
\lim _{h \searrow 0} H\left[\frac{X(t+h) \ominus X(t)}{h}, D_{H}^{g} X(t)\right]=0, \quad \lim _{h \searrow 0} H\left[\frac{X(t) \ominus X(t-h)}{h}, D_{H}^{g} X(t)\right]=0,
$$

or

(ii) for all $h>0$ sufficiently small, $\exists X(t) \ominus X(t+h), \exists X(t-h) \ominus X(t)$ and

$$
\lim _{h \searrow 0} H\left[\frac{X(t) \ominus X(t+h)}{-h}, D_{H}^{g} X(t)\right]=0, \quad \lim _{h \searrow 0} H\left[\frac{X(t-h) \ominus X(t)}{-h}, D_{H}^{g} X(t)\right]=0,
$$

or

(iii) for all $h>0$ sufficiently small, $\exists X(t+h) \ominus X(t), \exists X(t-h) \ominus X(t)$ and

$$
\lim _{h \searrow 0} H\left[\frac{X(t+h) \ominus X(t)}{h}, D_{H}^{g} X(t)\right]=0, \quad \lim _{h \searrow 0} H\left[\frac{X(t-h) \ominus X(t)}{-h}, D_{H}^{g} X(t)\right]=0,
$$


(iv) for all $h>0$ sufficiently small, $\exists X(t) \ominus X(t+h), \exists X(t) \ominus X(t-h)$ and the limits

$$
\lim _{h \searrow 0} H\left[\frac{X(t) \ominus X(t+h)}{-h}, D_{H}^{g} X(t)\right]=0, \quad \lim _{h \searrow 0} H\left[\frac{X(t) \ominus X(t-h)}{h}, D_{H}^{g} X(t)\right]=0 .
$$

( $h$ at denominators means $\frac{1}{h}$.) In this definition, case (i) ((i)-differentiability for short) corresponds to the classical $H$-derivative, so this differentiability concept is a generalization of the Hukuhara derivative. In this paper we consider only the first two of Definition 2.2. In the other cases, the derivative is trivial because it is reduced to a crisp element (more precisely, $D_{H}^{g} X(t) \in \mathbb{R}$ ). Further, we say that $X$ is (i)-differentiable or that we have (ii)-differentiation on $[a, b]$, if it is differentiable in the sense of (i) or (ii) of Definition 2.2, respectively.

Lemma 2.1 (see $[18,20,21])$ Assume that $F:\left[t_{0}, t_{0}+p\right] \times K_{C}(\mathbb{R}) \rightarrow K_{C}(\mathbb{R})$ is continuous. The interval-valued differential equation (1.1) is equivalent to one of the following integral equations:

$$
X(t)=X\left(t_{0}\right)+\int_{t_{0}}^{t} F(s, X(s)) d s, \quad \forall t \in\left[t_{0}, t_{0}+p\right]
$$

if $X$ is (i)-differentiable, and

$$
X(t)=X\left(t_{0}\right) \ominus(-1) \int_{t_{0}}^{t} F(s, X(s)) d s, \quad \forall t \in\left[t_{0}, t_{0}+p\right]
$$

if $X$ is (ii)-differentiable, provided that the H-difference exists.

Corollary 2.1 (see $[18,20,21]$ ) Let $X:\left[t_{0}, t_{0}+p\right] \rightarrow K_{C}(\mathbb{R})$ be given. Denote $X(t)=$ $[\underline{X}(t), \bar{X}(t)]$ for $t \in\left[t_{0}, t_{0}+p\right]$, where $\underline{X}, \bar{X}:\left[t_{0}, t_{0}+p\right] \rightarrow \mathbb{R}$.

(i) If the mapping $X$ is (i)-differentiable (i.e. classically Hukuhara differentiable) at $t \in\left[t_{0}, t_{0}+p\right]$, then the real-valued functions $\underline{X}, \bar{X}$ are differentiable at $t$ and $D_{H}^{g} X(t)=\left[\underline{X}^{\prime}(t), \bar{X}^{\prime}(t)\right]$.

(ii) If the mapping $X$ is (ii)-differentiable at $t \in\left[t_{0}, t_{0}+p\right]$, then the real-valued functions $\underline{X}, \bar{X}$ are differentiable at $t$ and $D_{H}^{g} X(t)=\left[\bar{X}^{\prime}(t), \underline{X}^{\prime}(t)\right]$.

The following comparison principle is fundamental in the investigation of the global existence of solutions of interval-valued functional differential equations.

Theorem 2.1 ([53]) Let $m \in C\left(\left[t_{0}-\sigma, \infty\right), \mathbb{R}\right)$ and satisfy the inequality

$$
D^{+} m(t) \leq g\left(t,\left|m_{t}\right|_{\sigma}\right), \quad t>t_{0}
$$

where $\left|m_{t}\right|_{\sigma}=\max _{-\sigma \leq s \leq 0}|m(t+s)|, g \in C\left(\left[t_{0}, \infty\right) \times \mathbb{R}^{+}, \mathbb{R}^{+}\right)$. Assume that $r(t)=r\left(t, t_{0}, u_{0}\right)$ is the maximal solution of the IVP

$$
\frac{d}{d t} u=g(t, u), \quad u\left(t_{0}\right)=u_{0} \geq 0
$$

existing on $\left[t_{0}, \infty\right)$. Then, if $\left|m_{t_{0}}\right|_{\sigma} \leq u_{0}$, we have $m(t) \leq r(t), t \in\left[t_{0}, \infty\right)$. 


\section{Inner product on space $\left(K_{C}(\mathbb{R}), H\right)$}

In the following, for $A, B \in K_{C}(\mathbb{R})$ we consider the function $\xi(\cdot ; A, B): \mathbb{R}^{+} \rightarrow \mathbb{R}$, defined by

$$
\xi(h ; A, B)=\frac{H[A+h B,\{0\}]-H[A,\{0\}]}{h} .
$$

Lemma 3.1 ([5]) For every $A, B \in K_{C}(\mathbb{R})$ the following limit exists

$$
[A, B]_{+}=\lim _{h \rightarrow 0^{+}} \frac{H[A+h B,\{0\}]-H[A,\{0\}]}{h} .
$$

In the following, for any $A, B \in K_{C}(\mathbb{R})$ we define the inner product product on $K_{C}(\mathbb{R})$ by

$$
(A, B)_{+}=\lim _{h \rightarrow 0^{+}} \frac{H^{2}(A+h B,\{0\})-H^{2}(A,\{0\})}{2 h} .
$$

From (3.3), we easily see that

$$
(A, B)_{+}=H[A,\{0\}][A, B]_{+} .
$$

Theorem 3.1 ([5]) The mappings $[\cdot, \cdot]_{+},(\cdot, \cdot)_{+}: K_{C}(\mathbb{R}) \times K_{C}(\mathbb{R}) \rightarrow \mathbb{R}$ are upper semicontinuous.

Lemma 3.2 For any $A, B \in K_{C}(\mathbb{R})$, the following properties hold:

(i) $\left|[A, B]_{+}\right| \leq H[B,\{0\}]$,

(ii) $\left|[A, B]_{+}-[A, C]_{+}\right| \leq H[B, C]$,

(iii) $[A, \lambda A]_{+}=\lambda H[A,\{0\}]$, for $\lambda \geq 0$,

(iv) $[k A, \lambda B]_{+}=\lambda H[B,\{0\}]$, for $k, \lambda \geq 0$,

(v) $[A, B+\lambda C]_{+} \leq[A, B]_{+}+\lambda H[C,\{0\}]$, for $\lambda \geq 0$.

Proof (i) For any $A, B \in K_{C}(\mathbb{R})$ and $h>0$, we have

$$
\begin{aligned}
\left|[A, B]_{+}\right| & =\left|\lim _{h \rightarrow 0^{+}} \xi(h ; A, B)\right|=\lim _{h \rightarrow 0^{+}} \frac{1}{h}|H[A+h B,\{0\}]-H[A,\{0\}]| \\
& \leq \lim _{h \rightarrow 0^{+}} \frac{1}{h} H[A+h B, A] .
\end{aligned}
$$

Passing to the limit when $h \rightarrow 0^{+}$, we get (i). Analogously, for $A, B, C \in K_{C}(\mathbb{R})$ and $h>0$, we obtain

$$
\left|[A, B]_{+}-[A, C]_{+}\right|=\lim _{h \rightarrow 0^{+}} \frac{1}{h}|H[h B,\{0\}]-H[h C,\{0\}]| .
$$

So, by passing to the limit when $h \rightarrow 0^{+}$, we get (ii). Equalities (iii), (iv), and (v) follow from (i).

Based on Lemma 3.2, we easily obtain the following properties for the inner product on $K_{C}(\mathbb{R})$.

Corollary 3.1 For any $A, B \in K_{C}(\mathbb{R})$, the following properties hold: 
(i) $\left|(A, B)_{+}\right| \leq H[A,\{0\}] H[B,\{0\}]$,

(ii) $(k A, \lambda B)_{+} \leq k \lambda H[A,\{0\}] H[B,\{0\}]$, for $k, \lambda \geq 0$,

(iii) $(k A, \lambda A)_{+} \leq k \lambda H^{2}(A,\{0\})$, for $k, \lambda \geq 0$,

(iv) $(A, B+C)_{+} \leq(A, B)_{+}+(A, C)_{+}$.

Theorem 3.2 If $X(\cdot):[a, b] \rightarrow K_{C}(\mathbb{R})$ is continuously differentiable on $[a, b]$, then $\|X(\cdot)\|=$ $H[X(\cdot),\{0\}]:[a, b] \rightarrow \mathbb{R}^{+}$is continuously differentiable on $[a, b]$ and

$$
\frac{d^{+}}{d t}\|X(t)\|=\left[X(t), D_{H}^{g} X(t)\right]_{+}
$$

for every $t \in[a, b]$.

Proof Since the way of the proof is similar for all four cases in Definition 2.2, we only consider the case of (i)-differentiability for $X$. Let $h>0$ be such that $t+h \in[a, b]$ is fixed. Let $m(t)=\|X(t)\|, t \in[a, b]$. Then we get

$$
\begin{aligned}
& \left|\frac{m(t+h)-m(t)}{h}-\frac{H\left[X(t)+h D_{H}^{g} X(t),\{0\}\right]-H[X(t),\{0\}]}{h}\right| \\
& \quad \leq \frac{1}{h} H\left[X(t+h), X(t)+h D_{H}^{g} X(t)\right] \\
& \quad=H\left[\frac{X(t+h) \ominus X(t)}{h}, D_{H}^{g} X(t)\right] .
\end{aligned}
$$

Since $\lim _{h \rightarrow 0^{+}} H\left[\frac{X(t+h) \ominus X(t)}{h}, D_{H}^{g} X(t)\right]=0$, we find that $\lim _{h \rightarrow 0^{+}} \frac{m(t+h)-m(t)}{h}$ exists and

$$
\lim _{h \rightarrow 0^{+}} \frac{m(t+h)-m(t)}{h}=\left[X(t), D_{H}^{g} X(t)\right]_{+} .
$$

The proof is complete.

Corollary 3.2 If $X(\cdot):[a, b] \rightarrow K_{C}(\mathbb{R})$ is continuously differentiable on $[a, b]$, then

$$
\frac{1}{2} \frac{d^{+}}{d t}\|X(t)\|^{2}=\left(X(t), D_{H}^{g} X(t)\right)_{+}
$$

for every $t \in[a, b]$.

Proof It is easy to prove this corollary with the result of Theorem 3.2 and (3.4). Indeed,

$$
\begin{aligned}
\frac{1}{2} \frac{d^{+}}{d t}\|X(t)\|^{2} & =\lim _{h \rightarrow 0^{+}} \frac{\|X(t+h)\|^{2}-\|X(t)\|^{2}}{2 h} \\
& =\lim _{h \rightarrow 0^{+}} \frac{(\|X(t+h)\|+\|X(t)\|)(\|X(t+h)\|-\|X(t)\|)}{2 h} \\
& =\|X(t)\| \lim _{h \rightarrow 0^{+}} \frac{\|X(t+h)\|-\|X(t)\|}{h}=\|X(t)\|\left[X(t), D_{H}^{g} X(t)\right]_{+} \\
& =\left(X(t), D_{H}^{g} X(t)\right)_{+} .
\end{aligned}
$$




\section{Global existence and uniqueness of solution of interval-valued functional differential equations}

For $\sigma>0$ let $C_{\sigma}=C\left([-\sigma, 0], K_{C}(\mathbb{R})\right)$ denote the space of continuous mappings from $[-\sigma, 0]$ to $K_{C}(\mathbb{R})$. Define a metric $H_{\sigma}$ in $C_{\sigma}$ by

$$
H_{\sigma}[X, Y]=\sup _{t \in[-\sigma, 0]} H[X(t), Y(t)]
$$

Let $p>0$. Denote $I=\left[t_{0}, t_{0}+p\right], J=\left[t_{0}-\sigma, t_{0}\right] \cup I=\left[t_{0}-\sigma, t_{0}+p\right]$. For any $t \in I$ denote by $X_{t}$ the element of $C_{\sigma}$ defined by $X_{t}(s)=X(t+s)$ for $s \in[-\sigma, 0]$.

Let us consider the interval-valued functional differential equations (IFDEs) with generalized Hukuhara derivative of the form

$$
\left\{\begin{array}{l}
D_{H}^{g} X(t)=F\left(t, X_{t}\right), \quad t \geq t_{0}, \\
X_{t_{0}}=\varphi_{0} \in C_{\sigma}
\end{array}\right.
$$

where $F \in C\left(I \times C_{\sigma}, K_{C}(\mathbb{R})\right)$, and the symbol $D_{H}^{g}$ denotes the generalized Hukuhara derivative from Definition 2.2. By a solution to (4.1) we mean an interval-valued mapping $X \in C\left(J, K_{C}(\mathbb{R})\right)$ that satisfies $X(t)=\varphi\left(t-t_{0}\right)$ for $t \in\left[t_{0}-\sigma, t_{0}\right]$, where $X$ is differentiable on $\left[t_{0}, t_{0}+p\right]$ and $D_{H}^{g} X(t)=F\left(t, X_{t}\right)$ for $t \in I$.

Lemma 4.1 ([6]) Assume that $F \in C\left(I \times C_{\sigma}, K_{C}(\mathbb{R})\right)$ and $X \in C\left(J, K_{C}(\mathbb{R})\right)$. Then the interval-valued mapping $t \rightarrow F\left(t, X_{t}\right)$ belongs to $C\left(I, K_{C}(\mathbb{R})\right)$.

Remark 4.1 ([6]) Under the assumptions of the lemma above we find that the mapping $t \rightarrow F\left(t, X_{t}\right)$ is integrable over the interval $I$.

Lemma 4.2 (cf. [6]) Assume that $F:\left[t_{0}, t_{0}+p\right] \times C_{\sigma} \rightarrow K_{C}(\mathbb{R})$ is continuous. An intervalvalued mapping $X: J \rightarrow K_{C}(\mathbb{R})$ is called a local solution to the problem (4.1) on J if and only if $X$ is a continuous interval-valued mapping and it satisfies one of the following intervalvalued integral equations:

(S1) $\left\{\begin{array}{l}X(t)=\varphi\left(t-t_{0}\right) \quad \text { for } t \in\left[t_{0}-\sigma, t_{0}\right], \\ X(t)=\varphi(0)+\int_{t_{0}}^{t} F\left(s, X_{s}\right) d s, \quad t \in I,\end{array}\right.$

if $X$ is (i)-differentiable.

(S2) $\left\{\begin{array}{l}X(t)=\varphi\left(t-t_{0}\right) \quad \text { for } t \in\left[t_{0}-\sigma, t_{0}\right], \\ X(t)=\varphi(0) \ominus(-1) \int_{t_{0}}^{t} F\left(s, X_{s}\right) d s, \quad t \in I,\end{array}\right.$

if $X$ is (ii)-differentiable.

Let us remark that in (4.3) is hidden the following statement: there exists a Hukuhara difference $\varphi(0) \ominus(-1) \int_{t_{0}}^{t} F\left(s, X_{s}\right) d s$.

Definition 4.1 Let $X: J \rightarrow K_{C}(\mathbb{R})$ be an interval-valued function which is (i)-differentiable. If $X$ and its derivative satisfy problem (4.1), we say $X$ is a (i)-solution of problem (4.1). An (i)-solution $X: J \rightarrow K_{C}(\mathbb{R})$ is unique if we have $H[X(t), Y(t)]=0$, for any $Y: J \rightarrow$ $K_{C}(\mathbb{R})$ which is a (i)-solution of (4.1). 
Definition 4.2 Let $X: J \rightarrow K_{C}(\mathbb{R})$ be an interval-valued function which is (ii)-differentiable. If $X$ and its derivative satisfy problem (4.1), we say that $X$ is a (ii)-solution of problem (4.1). An (ii)-solution $X: J \rightarrow K_{C}(\mathbb{R})$ is unique if we have $H[X(t), Y(t)]=0$, for any $Y: J \rightarrow$ $K_{C}(\mathbb{R})$ which is a (ii)-solution of (4.1).

Next, we shall establish the global existence and uniqueness result for IFDE (4.1). For the global existence and uniqueness we use the dissipative conditions. We now prove a comparison theorem, which is a useful tool in proving the global existence and uniqueness theorem.

Theorem 4.1 Assume that $F \in C\left(\mathbb{R}^{+} \times C_{\sigma}, K_{C}(\mathbb{R})\right)$ and $H[F(t, X), F(t, Y)] \leq g\left(t, H_{\sigma}[X, Y]\right)$ for $X, Y \in C_{\sigma}, t \in \mathbb{R}^{+}$, where $g \in C\left(\mathbb{R}^{+} \times \mathbb{R}^{+}, \mathbb{R}^{+}\right)$. Let $r\left(t, t_{0}, x_{0}\right)$ be the maximal solution of

$$
\left\{\begin{array}{l}
\frac{d x}{d t}=g(t, x) \\
x\left(t_{0}\right)=x_{0} \geq 0,
\end{array}\right.
$$

for $t \geq t_{0}$. Then, if $X(t), Y(t)$ are any (ii)-solutions (or (i)-solutions) of IFDE (4.1) such that $\varphi_{0}, \psi_{0} \in C_{\sigma}$ exist for $t \in \mathbb{R}^{+}$, we have $H[X(t), Y(t)] \leq r\left(t, t_{0}, x_{0}\right)$, provided that $H_{\sigma}\left[\varphi_{0}, \psi_{0}\right] \leq x_{0}$.

Proof We prove that for the case of (ii)-differentiability, the proof of the other case is similar. Since $X, Y$ are solutions of IFDE (4.1) and are (ii)-differentiable, we find that, for $h>0$ small enough, there exist Hukuhara differences $X(t-h) \ominus X(t), Y(t-h) \ominus Y(t)$. Now for $t \in \mathbb{R}^{+}$, set $m(t)=H[X(t), Y(t)]$, and we have

$$
\begin{aligned}
m(t-h)-m(t)= & H[X(t-h), Y(t-h)]-H[X(t), Y(t)] \\
\leq & H\left[X(t-h), X(t)+(-1) h F\left(t, X_{t}\right)\right] \\
& +H\left[X(t)+(-1) h F\left(t, X_{t}\right), Y(t)+(-1) h F\left(t, Y_{t}\right)\right] \\
& +H\left[Y(t)+(-1) h F\left(t, Y_{t}\right), Y(t-h)\right]-H[X(t), Y(t)] \\
\leq & H\left[X(t-h), X(t)+(-1) h F\left(t, X_{t}\right)\right] \\
& +H\left[Y(t)+(-1) h F\left(t, Y_{t}\right), Y(t-h)\right]+h H\left[F\left(t, X_{t}\right), F\left(t, Y_{t}\right)\right],
\end{aligned}
$$

from which we get

$$
\begin{aligned}
\frac{m(t-h)-m(t)}{h} \leq & H\left[\frac{X(t-h) \ominus X(t)}{-h}, F\left(t, X_{t}\right)\right]+H\left[F\left(t, Y_{t}\right), \frac{Y(t-h) \ominus Y(t)}{-h}\right] \\
& +H\left[F\left(t, X_{t}\right), F\left(t, Y_{t}\right)\right] .
\end{aligned}
$$

Taking $\lim \inf$ as $h \rightarrow 0^{+}$yields

$$
D^{-} m(t)=\lim _{h \rightarrow 0^{+}} \inf \frac{1}{h}[m(t-h)-m(t)] \leq g\left(t, H_{\sigma}[X, Y]\right)=g\left(t,\left|m_{t}\right|_{\sigma}\right),
$$

which together with the fact that $H_{\sigma}\left[\varphi_{0}, \psi_{0}\right] \leq x_{0}$, and by using Theorem 2.1, results in

$$
H[X(t), Y(t)] \leq r\left(t, t_{0}, x_{0}\right), \quad t \geq t_{0} .
$$

The proof is complete. 
Remark 4.2 Under the assumptions of Theorem 4.1 with $g(t, x)$ satisfying $g(t, x)=L \cdot x$, where $L>0$, we obtain $H[X(t), Y(t)] \leq H_{\sigma}\left[\varphi_{0}, \psi_{0}\right] \exp (L t)$.

Theorem 4.2 Assume that $F \in C\left(\mathbb{R}^{+} \times C_{\sigma}, K_{C}(\mathbb{R})\right), F$ maps bounded sets on to bounded sets and $F$ satisfies the following dissipative condition: there exists a continuous function $a: \mathbb{R}^{+} \rightarrow \mathbb{R}^{+}$and $r \geq 0$ such that

$$
\left(X(t), F\left(t, X_{t}\right)\right)_{+} \leq a(t) H^{2}[X,\{0\}]
$$

for every $t \geq 0$ and $X(t) \in K_{C}(\mathbb{R})$ with $H[X,\{0\}] \geq r$. Then:

(i) the interval-valued functional differential equation (4.1) has a (i)-solution on $\left[t_{0}, \infty\right)$;

(ii) the interval-valued functional differential equation (4.1) has a (ii)-solution on $\left[t_{0}, \infty\right.$ ) if the following conditions hold:

$$
\int_{t_{0}}^{t} \operatorname{len}\left(F\left(s, X_{s}\right)\right) d s \leq \operatorname{len}(\varphi(0)), \quad \forall t \geq t_{0} .
$$

Proof We prove that for the case of (i)-differentiability (i.e., case (i)), the proof of the other case is similar. For $\left(t_{0}, \varphi_{0}\right) \in \mathbb{R}^{+} \times C_{\sigma}$ fixed, we consider the sets $I=\left[t_{0}, t_{0}+p\right)$ and $B_{\rho}=\{X \in$ $\left.C_{\sigma}: H_{\sigma}[X,\{0\}] \leq \rho\right\}$, where $p>0$ and $\rho>0$. Since $F$ maps bounded sets onto bounded sets, we deduce that there exists $M>0$ such that $H\left[F\left(t, X_{t}\right),\{0\}\right] \leq M$, for every $(t, X) \in I \times B_{\rho}$. Therefore, there exists $\infty>\eta>t_{0}$ such that the problem (4.1) has at least locally a (i)solution $X(t)$ on some intervals $\left[t_{0}-\sigma, t_{0}+\eta\right]$. Let

$$
\mathcal{S}=\left\{X(t) \mid X(t) \text { is defined on } J_{X}=\left[t_{0}-\sigma, t_{0}+\eta_{X}\right) \text { and is the (i)-solution to (4.1) }\right\} \text {. }
$$

Then $\mathcal{S} \neq \emptyset$. Next, we define a partial order $\lesssim$ on $\mathcal{S}$ as follows: $X \lesssim Y$ if and only if $J_{X} \subseteq J_{Y}$ and $X(t)=Y(t)$ on $J_{X}$. Then standard application of Zorn's lemma assures the existence of the maximal element $Z$ in $(\mathcal{S}, \lesssim)$. The proof is complete if we show that $\eta_{Z}=\infty$. Suppose that it is not true, so that $\eta_{Z}<\infty$.

Step 1: We show that there exists $\gamma$ such that $H\left[Z\left(t_{0}, \varphi_{0}\right)(t),\{0\}\right] \leq \gamma$ for every $t \in J_{Z}$. Define $\|Z(t)\|=H\left[Z\left(t_{0}, \varphi_{0}\right)(t),\{0\}\right]$. Using Corollary 3.2 we have

$$
\frac{1}{2} \frac{d^{+}\|Z(t)\|^{2}}{d t}=\left(Z(t), D_{H}^{g} Z(t)\right)_{+}, \quad t \in J_{Z}
$$

which implies

$$
\frac{1}{2}\|Z(t)\|^{2}=\frac{1}{2}\left\|Z\left(t_{0}\right)\right\|^{2}+\int_{t_{0}}^{t}\left(Z(s), D_{H}^{g} Z(s)\right)_{+} d s, \quad t \in I_{Z},
$$

and so

$$
\frac{1}{2}\|Z(t)\|^{2}=\frac{1}{2}\left\|Z\left(t_{0}\right)\right\|^{2}+\int_{t_{0}}^{t}\left(Z(s), F\left(s, Z_{s}\right)\right)_{+} d s, \quad t \in J_{Z} .
$$

Now, we consider the sets $J_{1}=\left\{t \in J_{Z}:\|Z(t)\| \leq r\right\}$ and $J_{2}=J_{Z} \bigvee_{1}$. Then we have

$$
\left(Z(t), F\left(t, Z_{t}\right)\right)_{+} \leq a(t) H^{2}[Z,\{0\}]
$$


for every $t \in J_{2}$. On the other hand, since $F$ maps bounded sets onto bounded sets, there exists $M_{1}>0$ such that

$$
H\left[F\left(t, Z_{t},\right),\{0\}\right] \leq M_{1}, \quad \text { on } J_{Z} \times B_{\rho},
$$

and so, by Corollary 3.1, it follows that

$$
\left(Z(t), F\left(t, Z_{t}\right)\right)_{+} \leq\|Z(t)\| H\left[F\left(t, Z_{t}\right),\{0\}\right] \leq r M_{1}, \quad t \in J_{1} .
$$

From (4.7), (4.8) we obtain

$$
\left(Z(t), F\left(t, Z_{t}\right)\right)_{+} \leq r M_{1}+a(t) H^{2}[Z,\{0\}], \quad t \in J_{z},
$$

and so (4.6) becomes

$$
\frac{1}{2}\|Z(t)\|^{2} \leq\left(\frac{1}{2}\left\|Z\left(t_{0}\right)\right\|^{2}+r M_{1}\left(\eta_{Z}-t_{0}\right)\right)+\int_{t_{0}}^{t} a(s) H^{2}[Z(s),\{0\}] d s, \quad t \in J_{Z} .
$$

If we let $\xi(s)=H^{2}[Z(s),\{0\}]$, then we have

$$
\frac{1}{2} \xi(t) \leq e(t)+\int_{t_{0}}^{t} a(s) \xi(s) d s
$$

By Gronwall's lemma, from the inequality (4.9) we obtain

$$
\|Z(t)\|^{2} \leq \gamma^{2}=e(t)+\int_{t_{0}}^{t} e(s) a(s) \cdot \exp \left(\int_{s}^{t} a(r) d r\right) d s
$$

for every $t \in I_{Z}$. Here $e(t)=\left(\frac{1}{2}\left\|Z\left(t_{0}\right)\right\|^{2}+r M_{1}\left(\eta_{Z}-t_{0}\right)\right)$. Therefore, there exists $\gamma>0$ such that $H[Z(t),\{0\}] \leq \gamma$ on $J_{Z}$. We infer that $H\left[F\left(t, Z_{t}\right),\{0\}\right] \leq M_{Z}$, for every $t \in J_{Z}$.

Step 2: We show that $Z(\cdot)$ is Lipschitzian on $I_{Z}$. Indeed, for all $t_{1}, t_{2} \in I_{Z}$ with $t_{1} \leq t_{2}$, we have

$$
\begin{aligned}
H\left[Z\left(t_{0}, \varphi_{0}\right)\left(t_{1}\right), Z\left(t_{0}, \varphi_{0}\right)\left(t_{2}\right)\right] & =H\left[\varphi(0)+\int_{t_{0}}^{t_{1}} F\left(s, Z_{s}\right) d s, \varphi(0)+\int_{t_{0}}^{t_{2}} F\left(s, Z_{s}\right) d s\right] \\
& \leq H\left[\int_{t_{1}}^{t_{2}} F\left(s, Z_{s}\right) d s,\{0\}\right] \leq \int_{t_{1}}^{t_{2}} H\left[F\left(s, Z_{s}\right),\{0\}\right] d s \\
& \leq M_{Z}\left(t_{2}-t_{1}\right) .
\end{aligned}
$$

Therefore $Z(\cdot)$ has a continuous extension $Z^{*}(\cdot)$ on $\left[t_{0}, \eta_{Z}\right]$. By continuity, we have

$$
Z^{*}\left(\eta_{Z}\right)=\varphi(0)+\int_{t_{0}}^{\eta_{Z}} F\left(s, Z_{s}^{*}\right) d s
$$

This implies that $Z^{*}(\cdot)$ is a (ii)-solution of (4.1) on $\left[t_{0}-\sigma, \eta_{Z}\right]$. Further, we consider IFDE with the new initial function at $t=\eta_{Z}$

$$
D_{H}^{g} X=F\left(t, X_{t}\right), \quad X\left(\eta_{Z}\right)=\varphi\left(\eta_{Z}-t_{0}\right)=\psi_{0} .
$$


Then the last IFDE has a (ii)-solution $X^{*}(\cdot)$ on $\left[\eta_{Z}-\sigma, \eta_{Z}+q\right), q>0$. If we define

$$
Z_{1}(t)= \begin{cases}Z^{*}(t), & \text { for } t_{0}-\sigma \leq t \leq \eta_{Z} \\ Z^{*}(t), & \text { for } \eta_{Z}-\sigma \leq t \leq \eta_{Z}+q\end{cases}
$$

then it is clear that $Z_{1}(t)$ is a (ii)-solution of (4.1) on $\left[\eta_{Z}-\sigma, \eta_{Z}+q\right)$. This contradicts the maximality of $Z(t)$ and hence $\eta_{Z}=\infty$.

Theorem 4.3 Assume that the assumptions of Theorem 4.2 hold. In addition, we assume that $F \in C\left(\mathbb{R}^{+} \times C_{\sigma}, K_{C}(\mathbb{R})\right)$ and $H[F(t, X), F(t, Y)] \leq g\left(t, H_{\sigma}[X, Y]\right)$ for $X, Y \in C_{\sigma}, t \in \mathbb{R}^{+}$, where $g \in C\left(\mathbb{R}^{+} \times \mathbb{R}^{+}, \mathbb{R}^{+}\right), g(t, 0) \equiv 0$, and $x(t) \equiv 0$ is only a solution of

$$
\left\{\begin{array}{l}
\frac{d x}{d t}=g(t, x) \\
x\left(t_{0}\right)=0
\end{array}\right.
$$

for $t \geq t_{0}$. Then, for every $\left(t_{0}, \varphi_{0}\right) \in \mathbb{R}^{+} \times C_{\sigma}$, the interval-valued functional differential equation (4.1) has a unique solution on $\left[t_{0}, \infty\right)$ for each case.

Proof We prove it for the case of (ii)-differentiability, the proof of the other case is similar. Let $X$ and $Y$ be two (ii)-solutions of IFDE (4.1) and be (ii)-differentiable, then we find that, for $h>0$ small enough, there exist Hukuhara differences $X(t-h) \ominus X(t), Y(t-h) \ominus Y(t)$. Now for $t \in \mathbb{R}^{+}$, set $m(t)=H[X(t), Y(t)]$ and noting that $m\left(t_{0}\right)=0$, we get

$$
D^{-} m(t)=\lim _{h \rightarrow 0^{+}} \inf \frac{1}{h}[m(t-h)-m(t)] \leq g\left(t, H_{\sigma}\left[X_{t}, Y_{t}\right]\right),
$$

and so, by the comparison theorem for ordinary delay differential equations, it gives

$$
H[X(t), Y(t)] \leq r\left(t, t_{0}, 0\right), \quad t \geq t_{0},
$$

where $r\left(t, t_{0}, 0\right)$ is the solution of $(4.10)$ on $\left[t_{0}, \infty\right)$. Since by assumption $r\left(t, t_{0}, 0\right)=0$, we obtain $X(t)=Y(t)$ on $\left[t_{0}, \infty\right)$. The proof is complete.

Corollary 4.1 Under the assumptions of Theorem 4.2, if we suppose in addition that there exists $L>0$ such that

$$
H\left[F\left(t, X_{t}\right), F\left(t, Y_{t}\right)\right] \leq L H_{\sigma}[X, Y]
$$

on $\mathbb{R}^{+} \times C_{\sigma}$, then the IFDE (4.1) has a unique global solution for each case.

\section{Illustrations}

Let us consider again the interval-valued functional differential equation with the initial value condition

$$
\left\{\begin{array}{l}
D_{H}^{g} X(t)=F\left(t, X_{t}\right), \quad t \in I, \\
X(t)=\varphi\left(t-t_{0}\right), \quad t \in\left[t_{0}-\sigma, t_{0}\right],
\end{array}\right.
$$


where $F: I \times C_{\sigma} \rightarrow K_{C}(\mathbb{R})$ is a continuous interval-valued function and $\varphi \in C_{\sigma}$. We denote

$$
\begin{array}{ll}
X(t)=[\underline{X}(t), \bar{X}(t)], \quad t \geq t_{0}, \\
X(t)=[\underline{\varphi}(t), \bar{\varphi}(t)], \quad t \in\left[t_{0}-\sigma, t_{0}\right],
\end{array}
$$

and

$$
F\left(t, X_{t}\right)=\left[\underline{F}\left(t, \underline{X}_{t}, \bar{X}_{t}\right), \bar{F}\left(t, \underline{X}_{t}, \bar{X}_{t}\right)\right], \quad t \geq t_{0} .
$$

In (5.1), we shall solve it by two types of Hukuhara derivative, which are defined in Definition 2.2. Consequently, based on the type of differentiability, we have the following two cases.

If we consider the derivative of $X(t)$ by using (i)-differentiability, then from Corollary 2.1, we have $D_{H}^{g} X(t)=\left[\underline{X}^{\prime}(t), \bar{X}^{\prime}(t)\right]$, for $t \geq t_{0}$. Therefore, (5.1) is translated into the following delay differential system:

$$
\left\{\begin{array}{l}
\underline{X}^{\prime}(t)=\underline{F}\left(t, \underline{X}_{t}, \bar{X}_{t}\right), \quad t \geq t_{0}, \\
\bar{X}^{\prime}(t)=\bar{F}\left(t, \underline{X}_{t}, \bar{X}_{t}\right), \quad t \geq t_{0}, \\
\underline{\bar{X}}(t)=\underline{\varphi}\left(t-t_{0}\right), \quad t_{0}-\sigma \leq t \leq t_{0}, \\
\bar{\varphi}\left(t-t_{0}\right), \quad t_{0}-\sigma \leq t \leq t_{0} .
\end{array}\right.
$$

If we consider the derivative of $X(t)$ by using (ii)-differentiability, then from Corollary 2.1, we have $D_{H}^{g} X(t)=\left[\bar{X}^{\prime}(t), \underline{X}^{\prime}(t)\right]$, for $t \geq t_{0}$. Therefore, (5.1) is translated into the following delay differential system:

$$
\left\{\begin{array}{l}
\bar{X}^{\prime}(t)=\underline{F}\left(t, \underline{X}_{t}, \bar{X}_{t}\right), \quad t \geq t_{0}, \\
\underline{X}^{\prime}(t)=\bar{F}\left(t, \underline{X}_{t}, \bar{X}_{t}\right), \quad t \geq t_{0}, \\
\underline{X}(t)=\underline{\varphi}\left(t-t_{0}\right), \quad t_{0}-\sigma \leq t \leq t_{0}, \\
\bar{X}(t)=\bar{\varphi}\left(t-t_{0}\right), \quad t_{0}-\sigma \leq t \leq t_{0} .
\end{array}\right.
$$

Remark 5.1 If we ensure that the solutions $(\underline{X}(t), \bar{X}(t))$ of the systems (5.2) and (5.3), respectively, are valid sets of interval-valued functions and if the derivatives $\left(\underline{X}^{\prime}(t), \bar{X}^{\prime}(t)\right)$ are valid sets of interval-valued functions with two kinds of differentiability, respectively, then we can construct the solution of the interval-valued functional differential equation (5.1).

Next, we shall consider some examples being simple illustrations of the theory of interval-valued functional differential equations.

Example 5.1 Let us consider the linear interval-valued functional differential equation under two kinds Hukuhara derivative,

$$
\left\{\begin{array}{l}
D_{H}^{g} X(t)=-\lambda X\left(t-\frac{1}{2}\right), \\
X(t)=\varphi(t), \quad t \in\left[-\frac{1}{2}, 0\right],
\end{array}\right.
$$

where $\varphi(t)=[-1,1], \lambda>0$. In this example we shall solve $(5.4)$ on $[0,1]$. 


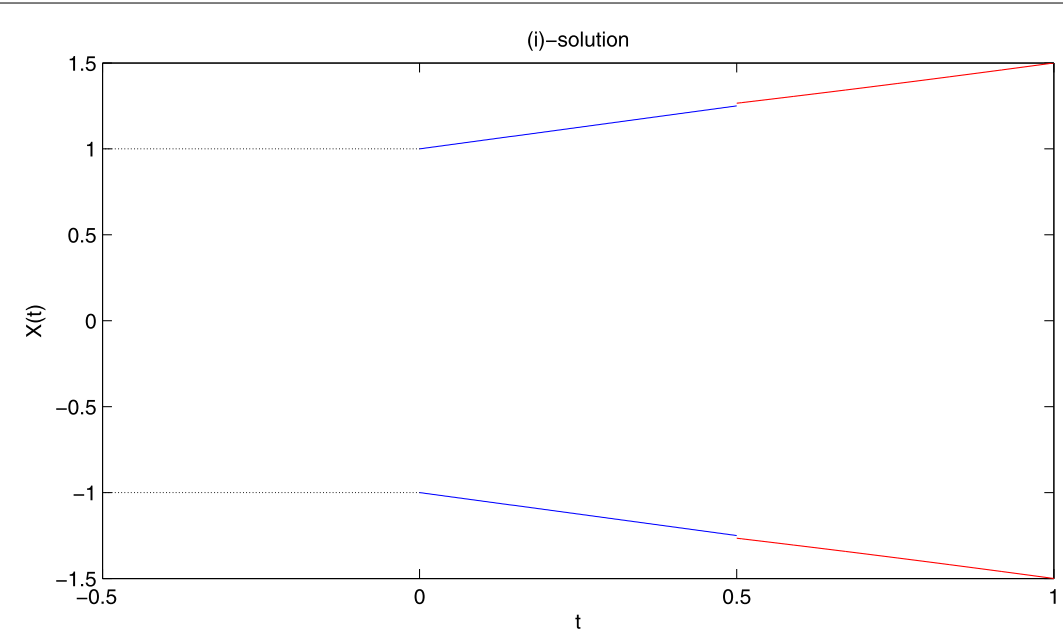

Figure 1 (i)-solution to $(5.4)(\lambda=0.5)$.

Case 1: Considering (i)-differentiability, problem (5.4) is translated into the following delay system:

$$
\left\{\begin{array}{l}
\underline{X}^{\prime}(t)=-\lambda \bar{X}\left(t-\frac{1}{2}\right), \quad t \geq 0, \\
\bar{X}^{\prime}(t)=-\lambda \underline{X}\left(t-\frac{1}{2}\right), \quad t \geq 0, \\
\underline{X}(t)=-1, \quad-\frac{1}{2} \leq t \leq 0, \\
\bar{X}(t)=1, \quad-\frac{1}{2} \leq t \leq 0 .
\end{array}\right.
$$

Solving delay system (5.5) by using the method of steps, we obtain a unique (i)-solution to (5.4) defined on $[0,1]$ and it is of the form

$$
X(t)= \begin{cases}{[-1(1+\lambda t),(1+\lambda t)]} & \text { for } t \in\left[0, \frac{1}{2}\right] \\ {\left[-1\left(1+\lambda t+\frac{\lambda^{2}(t-1)^{2}}{2}\right), 1+\lambda t+\frac{\lambda^{2}(t-1)^{2}}{2}\right]} & \text { for } t \in\left[\frac{1}{2}, 1\right] .\end{cases}
$$

The (i)-solution is illustrated in Figure 1.

Case 2: Considering (ii)-differentiability, problem (5.4) is translated into the following delay system:

$$
\left\{\begin{array}{l}
\bar{X}^{\prime}(t)=-\lambda \bar{X}\left(t-\frac{1}{2}\right), \quad t \geq 0, \\
\underline{X}^{\prime}(t)=-\lambda \underline{X}\left(t-\frac{1}{2}\right), \quad t \geq 0, \\
\underline{X}(t)=-1, \quad-\frac{1}{2} \leq t \leq 0, \\
\bar{X}(t)=1, \quad-\frac{1}{2} \leq t \leq 0 .
\end{array}\right.
$$

We obtain a unique (ii)-solution to (5.4) defined on $[0,1]$ and it is of the form

$$
X(t)= \begin{cases}{[\lambda t-1,1-\lambda t]} & \text { for } t \in\left[0, \frac{1}{2}\right], \\ {\left[-1\left(1-\lambda t+\frac{\lambda^{2}(t-1)^{2}}{2}\right), 1-\lambda t+\frac{\lambda^{2}(t-1)^{2}}{2}\right]} & \text { for } t \in\left[\frac{1}{2}, 1\right] .\end{cases}
$$

The (ii)-solution is illustrated in Figure 2. 


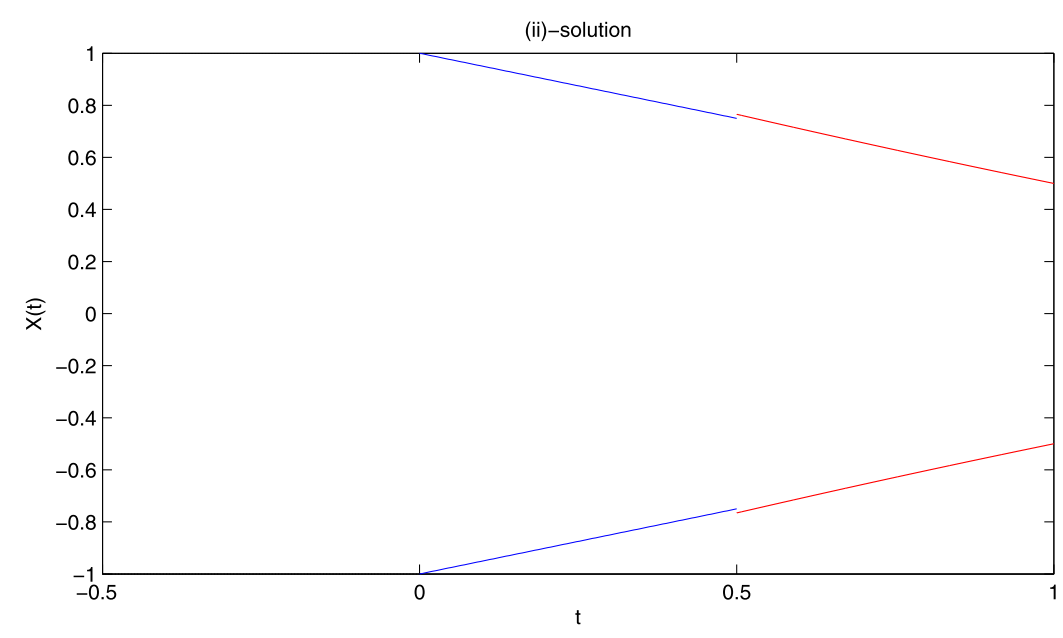

Figure 2 (ii)-solution to $(5.4)(\lambda=0.5)$.

Example 5.2 Let us consider the linear interval-valued functional differential equation under two kinds of Hukuhara derivative,

$$
\left\{\begin{array}{l}
D_{H}^{g} X(t)=\lambda X(t-d), \\
X(t)=\varphi(t), \quad t \in[-d, 0],
\end{array}\right.
$$

where $\varphi(t)=[4-t, 6-t], t \in[-d, 0], d>0, \lambda>0$. In this example we shall solve (5.7) on $[0, d]$.

One can obtain the (i)-solution and (ii)-solution by using the methods as in the above examples.

The (i)-solution to (5.7) defined on $[0, d]$ and it is of the form

$$
X(t)=\left[\lambda t\left[4-\frac{t}{2}+d\right]+4, \lambda t\left[6-\frac{t}{2}+d\right]+6\right]
$$

The (ii)-solution to (5.7) is defined on $[0, d]$ and it is of the form

$$
X(t)=\left[\lambda t\left[6-\frac{t}{2}+d\right]+4, \lambda t\left[4-\frac{t}{2}+d\right]+6\right] .
$$

In Figures 3 and 4, the (i)-solution and (ii)-solution curves of (5.7) are given.

Example 5.3 Consider the interval-valued functional differential equation

$$
\left\{\begin{array}{l}
D_{H}^{g} X(t)=\frac{\lambda}{1+t^{2}} X\left(t-\frac{1}{2}\right), \quad t \geq 0 \\
X(t)=\left[\frac{1}{1+t}, \frac{3}{1+t}\right], \quad t \in\left[-\frac{1}{2}, 0\right]
\end{array}\right.
$$

where $F:[0, \infty) \times C_{\sigma} \rightarrow K_{C}(\mathbb{R})$ is given by $F\left(t, X_{t}\right)=\frac{1}{1+t^{2}} X\left(t-\frac{1}{2}\right)$ and $\varphi(t)=\left[\frac{1}{1+t}, \frac{3}{1+t}\right]$, $t \in\left[-\frac{1}{2}, 0\right], \lambda \in[-1,1] \backslash\{0\}$. 


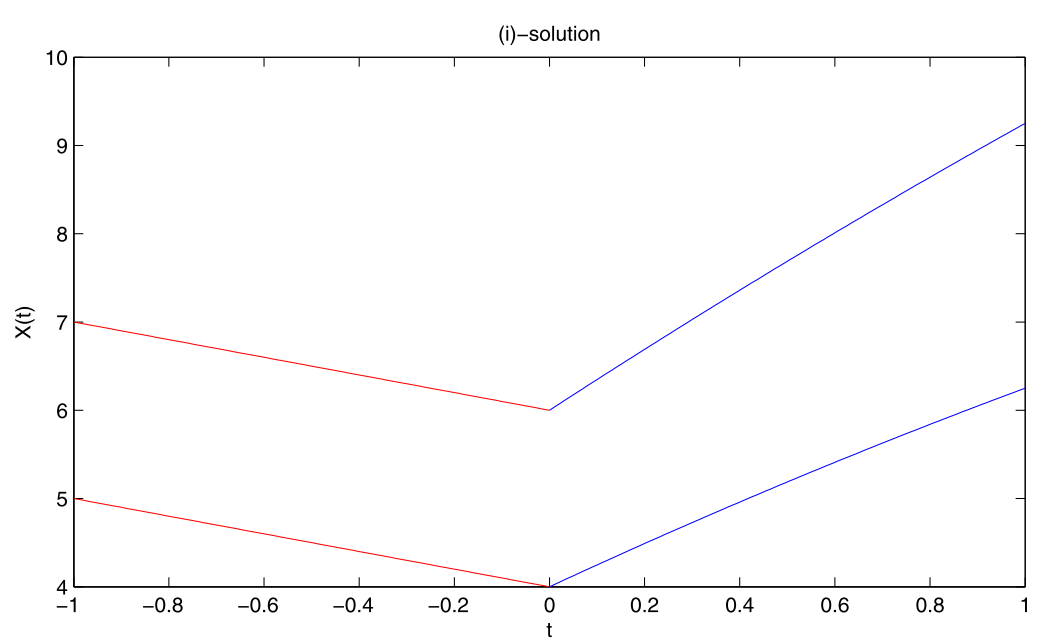

Figure 3 (i)-solution to (5.7) $(\lambda=0.5, d=1)$.

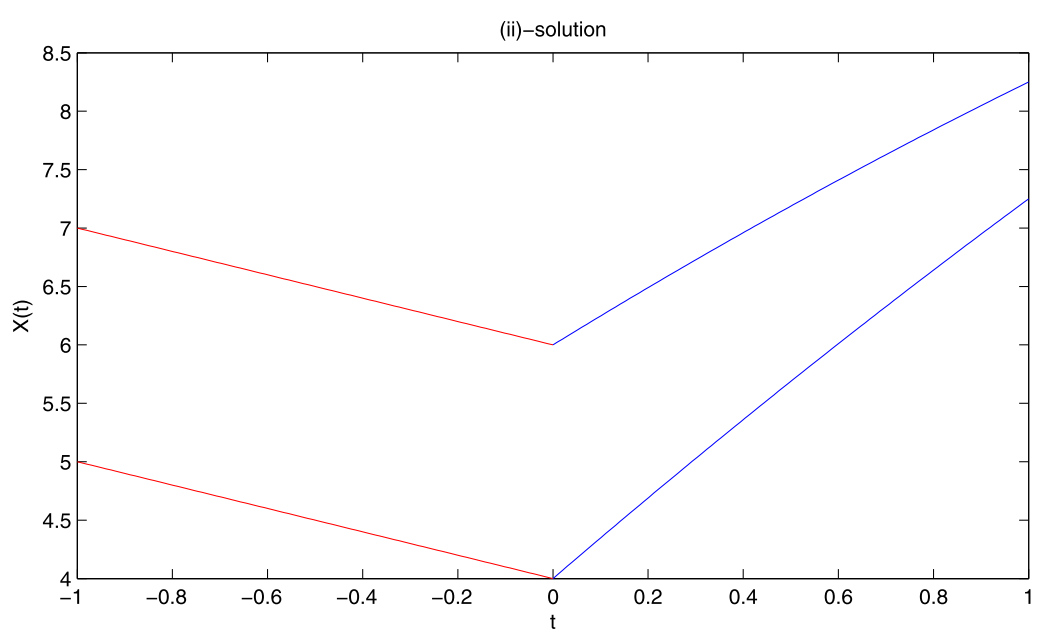

Figure 4 (ii)-solution to (5.7) $(\lambda=0.5, d=1)$.

Case 1: Consider $\lambda \in(0,1]$. If we consider $D_{H}^{g} X(t)$ in the sense of (i)-differentiability, we have to solve the following delay differential system:

$$
\left\{\begin{array}{l}
\underline{X}^{\prime}(t)=\frac{\lambda}{1+t^{2}} \underline{X}\left(t-\frac{1}{2}\right), \quad t \geq 0, \\
\overline{\bar{X}}^{\prime}(t)=\frac{\lambda}{1+t^{2}} \bar{X}\left(t-\frac{1}{2}\right), \quad t \geq 0, \\
\underline{X}(t)=\frac{1}{1+t}, \quad \bar{X}(t)=\frac{3}{1+t}, \quad-\frac{1}{2} \leq t \leq 0 .
\end{array}\right.
$$

Following the method of steps, we can obtain the expression of the (i)-solution on $[0,1]$, which is represented in Figure 5.

On the other hand, if $D_{H}^{g} X(t)$ is (ii)-differentiable, then we have to solve the following delay differential system: 


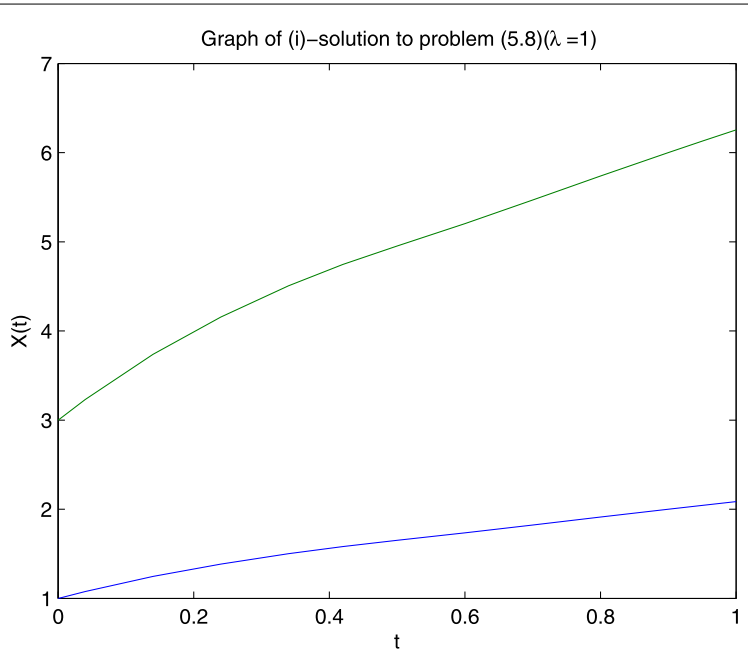

Figure 5 (i)-solution to $(5.8)(\lambda=1)$.

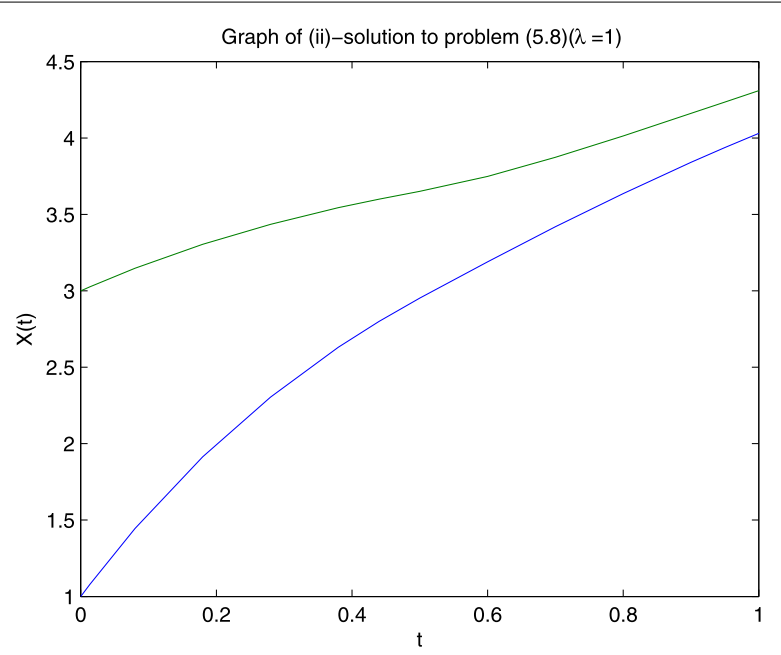

Figure 6 (ii)-solution to $(5.8)(\lambda=1)$.

$$
\left\{\begin{array}{l}
\underline{X}^{\prime}(t)=\frac{\lambda}{1+t^{2}} \bar{X}\left(t-\frac{1}{2}\right), \quad t \geq 0, \\
\overline{\bar{X}}^{\prime}(t)=\frac{\lambda}{1+t^{2}} \underline{X}\left(t-\frac{1}{2}\right), \quad t \geq 0, \\
\underline{X}(t)=\frac{1}{1+t}, \quad \bar{X}(t)=\frac{3}{1+t}, \quad-\frac{1}{2} \leq t \leq 0 .
\end{array}\right.
$$

By the method of steps, we can obtain the expression of the (ii)-solution on $[0,1]$ corresponding to the initial condition $\varphi(t)$. This solution is illustrated in Figure 6 .

Case 2: Consider $\lambda \in[-1,0)$. If we consider $D_{H}^{g} X(t)$ in the form where it is (i)differentiable, we have to solve the following delay differential system:

$$
\left\{\begin{array}{l}
\underline{X}^{\prime}(t)=\frac{\lambda}{1+t^{2}} \bar{X}\left(t-\frac{1}{2}\right), \quad t \geq 0, \\
\overline{\bar{X}}^{\prime}(t)=\frac{\lambda}{1+t^{2}} \underline{X}\left(t-\frac{1}{2}\right), \quad t \geq 0, \\
\underline{X}(t)=\frac{1}{1+t}, \quad \bar{X}(t)=\frac{3}{1+t}, \quad-\frac{1}{2} \leq t \leq 0 .
\end{array}\right.
$$

This solution is shown in Figure 7. 


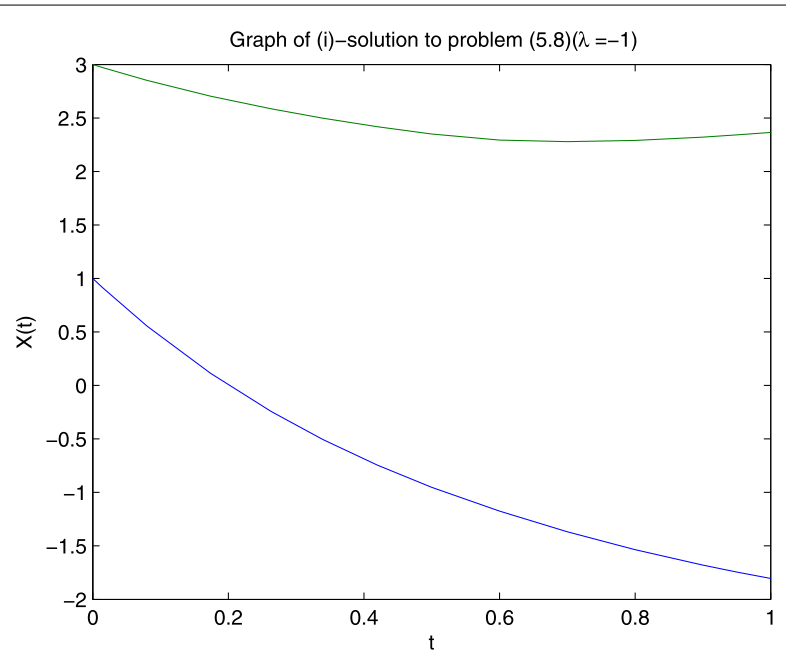

Figure 7 (i)-solution to $(5.8)(\lambda=-1)$.

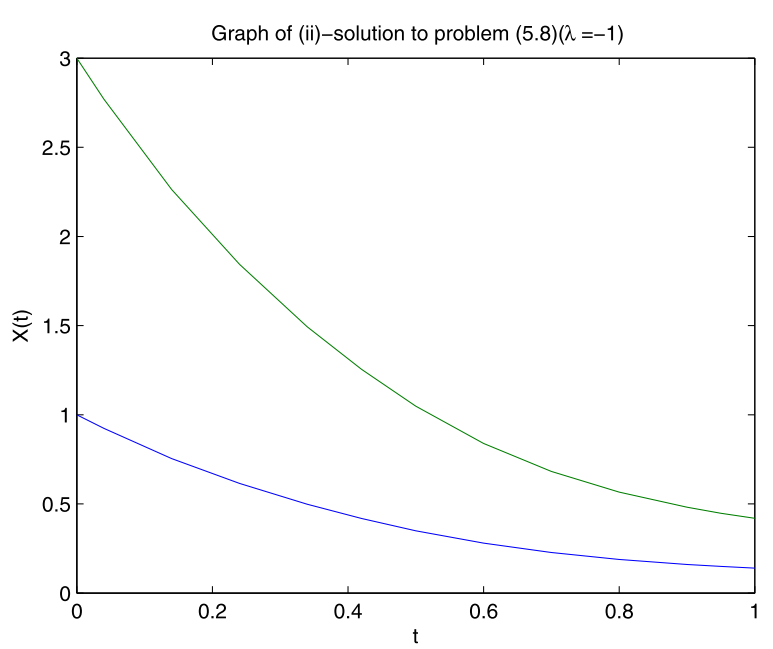

Figure 8 (ii)-solution to $(5.8)(\lambda=-1)$.

If we consider $D_{H}^{g} X(t)$ in the sense of (ii)-differentiability, we have to solve the following delay differential system:

$$
\left\{\begin{array}{l}
\underline{X}^{\prime}(t)=\frac{\lambda}{1+t^{2}} \underline{X}\left(t-\frac{1}{2}\right), \quad t \geq 0, \\
\overline{\bar{X}}^{\prime}(t)=\frac{\lambda}{1+t^{2}}\left(t-\frac{1}{2}\right), \quad t \geq 0, \\
\underline{X}(t)=\frac{1}{1+t}, \quad \bar{X}(t)=\frac{3}{1+t}, \quad-\frac{1}{2} \leq t \leq 0 .
\end{array}\right.
$$

The (ii)-solution is illustrated in Figure 8.

\section{Competing interests}

The authors declare that they have no competing interests. 


\section{Author details}

${ }^{1}$ Faculty of Mathematics and Computer Science, University of Science, VNU, Ho Chi Minh City, Vietnam. ${ }^{2}$ Faculty of Foundation Sciences, University of Technical Education, Ho Chi Minh City, Vietnam. ${ }^{3}$ Division of Computational Mathematics and Engineering, Institute for Computational Science, Ton Duc Thang University, Ho Chi Minh City, Vietnam.

\section{Acknowledgements}

The authors would like to express their gratitude to the anonymous referees for their helpful comments and suggestions, which have greatly improved the paper. The second named author would like to thank the University of Technical Education, Ho Chi Minh City, Vietnam.

\section{Received: 23 November 2013 Accepted: 13 June 2014 Published: 23 Jul 2014}

\section{References}

1. Ahmad, B, Sivasundaram, S: Dynamics and stability of impulsive hybrid setvalued integro-differential equations with delay. Nonlinear Anal., Theory Methods Appl. 65, 2082-2093 (2006)

2. Hale, JK: Theory of Functional Differential Equations. Springer, New York (1977)

3. Kuang, Y: Delay Differential Equations: With Applications in Population Dynamics. Academic Press, Boston (1993)

4. Lupulescu, V: On a class of fuzzy functional differential equations. Fuzzy Sets Syst. 160, 1547-1562 (2009)

5. Lupulescu, V: Initial value problem for fuzzy differential equations under dissipative conditions. Inf. Sci. 178, 4523-4533 (2008)

6. Malinowski, MT: Second type Hukuhara differentiable solutions to the delay set-valued differential equations. Appl. Math. Comput. 218, 9427-9437 (2012)

7. Kolmanovskii, VB, Myshkis, A: Applied Theory of Functional Differential Equations. Kluwer Academic, Dordrecht (1992)

8. De Blasi, FS, lervolino, F: Equazioni differenziali con soluzioni a valore compatto convesso. Boll. Unione Mat. Ital. 4, 194-501 (1969)

9. Agarwal, RP, Arshad, S, O'Regan, D, Lupulescu, V: A Schauder fixed point theorem in semilinear spaces and applications. Fixed Point Theory Appl. 2013, Article ID 306 (2013)

10. Agarwal, RP, O'Regan, D, Lakshmikantham, V: Viability theory and fuzzy differential equations. Fuzzy Sets Syst. 151, 563-580 (2005)

11. Agarwal, RP, O'Regan, D, Lakshmikantham, V: A stacking theorem approach for fuzzy differential equations. Nonlinear Anal. TMA 55, 299-312 (2003)

12. Abbas, $U$, Lupulescu, V: Set functional differential equations. Commun. Appl. Nonlinear Anal. 18, 97-110 (2011)

13. Hoa, NV, Phu, ND: On maximal and minimal solutions for set-valued differential equations with feedback control. Abstr. Appl. Anal. 2012, Article ID 816218 (2012). doi:10.1155/2012/816218

14. Devi, JV: Generalized monotone iterative technique for set differential equations involving causal operators with memory. Int. J. Adv. Eng. Sci. Appl. Math. (2011). doi:10.1007/s12572-011-0031-1

15. Lakshmikantham, V, Bhaskar, TG, Devi, JV: Theory of Set Differential Equations in Metric Spaces. Cambridge Scientific Publisher, Cambridge (2006)

16. Lakshmikantham, $\mathrm{V}$, Tolstonogov, AA: Existence and interrelation between set and fuzzy differential equations. Nonlinear Anal. TMA 55, 255-268 (2003)

17. Lupulescu, $V:$ Hukuhara differentiability of interval-valued functions and interval differential equations on time scales. Inf. Sci. 248, 50-67 (2013)

18. Stefanini, L, Bede, B: Generalized Hukuhara differentiability of interval-valued functions and interval differential equations. Nonlinear Anal. TMA 71, 1311-1328 (2009)

19. Lupulescu, V: Successive approximations to solutions of set differential equations in Banach spaces. Dyn. Contin. Discrete Impuls. Syst. Ser. A Math. Anal. 15, 391-401 (2008)

20. Malinowski, MT: Interval Cauchy problem with a second type Hukuhara derivative. Inf. Sci. 213, 94-105 (2012)

21. Malinowski, MT: Interval differential equations with a second type Hukuhara derivative. Appl. Math. Lett. 24, 2118-2123 (2011)

22. Hukuhara, M: Intégration des applications mesurables dont la valeur est un compact convex. Funkc. Ekvacioj 10, 205-229 (1967)

23. Agarwal, RP, O'Regan, D: Existence for set differential equations via multivalued operator equations. In: Differential Equations and Applications, vol. 5, pp. 1-5. Nova Publ., New York (2007)

24. Bhaskar, TG, Lakshmikantham, V: Set differential equations and flow invariance. J. Appl. Anal. 82, 357-368 (2003)

25. Bhaskar, TG, Lakshmikantham, V, Devi, JV: Nonlinear variation of parameters formula for set differential equations in a metric space. Nonlinear Anal. 63, 735-744 (2005)

26. Lakshmikantham, V, Vatsala, AS: Set differential equations and monotone flows. Nonlinear Dyn. Syst. Theory 3 , 151-161 (2003)

27. Bhaskar, TG, Devi, JV: Stability criteria in set differential equations. Math. Comput. Model. 41, 1371-1378 (2005)

28. Ahmada, B, Sivasundaram, S: The monotone iterative technique for impulsive hybrid set valued integro-differential equations. Nonlinear Anal. 65, 2260-2276 (2006)

29. Quang, LT, Phu, ND, Hoa, NV, Vu, H: On the maximal and minimal solutions for set integro-differential equations with feedback control. Nonlinear Stud. 20, 39-56 (2013)

30. An, TV, Hoa, NV, Phu, ND: Global existence of solutions for interval-valued integro-differential equations under generalized H-differentiability. Adv. Differ. Equ. 2013, Article ID 217 (2013). doi:10.1186/1687-1847-2013-217

31. Hoa, NV, An, TV, Phu, ND: A note on solutions of interval-valued Volterra integral equations. J. Integral Equ. Appl. 26, 1-14 (2014)

32. Hoa, NV, Phu, ND: Global existence of solutions for interval-valued second-order differential equations under generalized Hukuhara derivative. Adv. Differ. Equ. 2013, Article ID 290 (2013). doi:10.1186/1687-1847-2013-290

33. Bede, B, Gal, SG: Generalizations of the differentiability of fuzzy-number-valued functions with applications to fuzzy differential equations. Fuzzy Sets Syst. 151, 581-599 (2005)

34. Bede, B, Rudas, IJ, Bencsik, AL: First order linear fuzzy differential equations under generalized differentiability. Inf. Sci. $177,1648-1662(2007)$ 
35. Bede, B, Stefanini, L: Generalized differentiability of fuzzy-valued functions. Fuzzy Sets Syst. 230, 119-141 (2013)

36. Bede, B: A note on 'Two-point boundary value problems associated with non-linear fuzzy differential equations'. Fuzzy Sets Syst. 157, 986-989 (2006)

37. Hoa, NV, Phu, ND: Fuzzy functional integro-differential equations under generalized H-differentiability. J. Intell. Fuzzy Syst. 26, 2073-2085 (2014)

38. Vu, H, Dong, LS, Hoa, NV: Random fuzzy functional integro-differential equations under generalized Hukuhara differentiability. J. Intell. Fuzzy Syst. (2013, accepted). doi:10.3233/IFS-131116

39. Hoa, NV, Tri, PV, Dao, TT: Some global existence results and stability theorem for fuzzy functional differential equations. J. Intell. Fuzzy Syst. (2013, accepted)

40. Tri, PV, Hoa, NV, Phu, ND: Sheaf fuzzy problems for functional differential equations. Adv. Differ. Equ. 2014, Article ID $156(2014)$

41. Malinowski, MT: Random fuzzy differential equations under generalized Lipschitz condition. Nonlinear Anal., Real World Appl. 13, 860-881 (2012)

42. Malinowski, MT: Existence theorems for solutions to random fuzzy differential equations. Nonlinear Anal., Theory Methods Appl. 73, 1515-1532 (2010)

43. Malinowski, MT: On random fuzzy differential equations. Fuzzy Sets Syst. 160, 3152-3165 (2009)

44. Song, S, Wu, C: Existence and uniqueness of solutions to Cauchy problem of fuzzy differential equations. Fuzzy Sets Syst. 110, 55-67 (2000)

45. Vu, H, Hoa, NV, Phu, ND: The local existence of solutions for random fuzzy integro-differential equations under generalized H-differentiability. J. Intell. Fuzzy Syst. (2013). doi:10.3233/IFS-130940

46. Malinowski, MT: Strong solutions to stochastic fuzzy differential equations of Itô type. Math. Comput. Model. 55 918-928 (2012)

47. Malinowski, MT: Itô type stochastic fuzzy differential equations with delay. Syst. Control Lett. 61, 692-701 (2012)

48. Malinowski, MT: Some properties of strong solutions to stochastic fuzzy differential equations. Inf. Sci. 252, 62-80 (2013)

49. Malinowski, MT: Approximation schemes for fuzzy stochastic integral equations. Appl. Math. Comput. 219 11278-11290 (2013)

50. Malinowski, MT: On a new set-valued stochastic integral with respect to semimartingales and its applications. J. Math Anal. Appl. 408, 669-680 (2013)

51. Malinowski, MT: Modeling with stochastic fuzzy differential equations. In: Chakraverty, S (ed.) Mathematics of Uncertainty Modeling in the Analysis of Engineering and Science Problems, pp. 150-172. IGI Global, Hershey (2014)

52. Malinowski, MT: On set differential equations in Banach spaces - a second type Hukuhara differentiability approach. Appl. Math. Comput. 219, 289-305 (2012)

53. Lakshmikantham, V: Theory of fractional functional differential equations. Nonlinear Anal., Theory Methods Appl. 69 3337-3343 (2008)

10.1186/1687-1847-2014-198

Cite this article as: Phu et al.: Interval-valued functional differential equations under dissipative conditions. Advances in Difference Equations 2014, 2014:198

\section{Submit your manuscript to a SpringerOpen ${ }^{\circ}$ journal and benefit from:}

- Convenient online submission

- Rigorous peer review

- Immediate publication on acceptance

- Open access: articles freely available online

- High visibility within the field

- Retaining the copyright to your article 